\title{
Reliability-based design algorithm for artificially replaced hip prosthesis considering material property uncertainty
}

\author{
Kharmanda Ghias \\ Department of Biomedical Engineering, Lund University, \\ Lund, Sweden \\ Ghias.Kharmanda@bme.lth.se
}

\author{
Antypas Imad Rezakalla \\ Department of design principles of machines, \\ Don State Technical University \\ Rostov-on-Don, Russia \\ imad.antypas@mail.ru
}

\begin{abstract}
The material properties of living tissue are subjected to uncertainty according to several factors such as ageing, disease, nutrition ... For example; the bone is a composite structure containing organic and inorganic components. Inorganic components are essentially responsible for the compression strength and stiffness, while organic components provide the corresponding tension properties. A mathematical formulation representing the relationship between compression strength and material properties of the different bone layers is developed. This formulation is next integrated into a reliability algorithm in order to assess the reliability level of the implantbone study. This way the optimization process is executed in a non-deterministic space (iteratively changed). However, the efficiency of the proposed algorithm is demonstrated on the identification of the failure mode (scenario) in the artificially replaced hip prosthesis. It also allows us extending to multiple failure modes. The proposed algorithm reduces the computing time consumption and provides high convergence stability levels. Numerical applications are carried out in order to show the efficiency of the proposed algorithm considering several daily loading cases on a hip prosthesis.
\end{abstract}

Keywords-material properties; reliability analysis; failure modes, artificially replaced hip prosthesis

\section{INTRODUCTION}

In the classical design optimization methods for implantbone studies, a safety and satisfactory performance cannot completely be guarantee, due in part to the randomness of different bone properties, geometry and loading. A number of uncertainties are encountered during the design of hip prosthesis. Some researchers have developed computational tools using a set of random variables in context of probabilistic analysis and durability studies [1,2]. In the reliability context, the previous works considered the loading uncertainty [3]. In this work, the material properties of the different bone layers are considered as uncertain parameters. So their corresponding yield stress values will not be deterministic, that leads to integrate variable limitations into the optimization process. In general, three kinds of variables can be classified:
1) Design variables $x_{i}:$ The design variables are deterministic variables defined in order to optimize the system. They represent control parameters of the mechanical system (e.g., dimensions, materials, loads) and of the probabilistic model (e.g., mean values and/or standarddeviations of random variables),

2) Random variables $y_{i}$ : The uncertainties are modeled by stochastic physical variables affecting the failure scenario. These variables can represent geometrical dimensions, material characteristics or applied external loading. The knowledge of these variables is not, at best, more than statistical information and it can be admitted as a representation in the form of random variables. The random physical variables represent the structural uncertainties, which are identified by probabilistic distributions,

3) Normalized variables $u_{i}$ : They represent the transformation of the random variables from the physical space to a normalized one according to certain probabilistic distribution laws [4].

ANSYS software is an efficient simulation and optimization tool for structural designers. However, there is no module for reliability analysis in this software. In addition, the artificially hip replacement joint represents a complicated design problem where the constraints are variables according to ageing, disease, nutrition ... According to Keller's model [5], the yield stress of bone is related with the bone density that leads to variable boundaries, but in ANSYS software the boundaries for constraints should be fixed. So, there is a strong need to develop a new algorithm for reliability problem with variable boundaries. In this work, a new strategy consists in implementing the reliability analysis into ANSYS software using APDL (ANSYS Parametric Design Language) technology is presented. The uncertainty integration of living tissue material properties can be considered as a novel aspect. The algorithm controls automatically the yield stress relating with the bone stiffness. 


\section{MODEL AND DEVELOPMENTS}

\section{A. Implant-bone model}

In realistic $3 \mathrm{D}$ model, the stem is surrounded by bone layers with a complex modeling shape. Fig. 1a shows a 3D model of the studied stem, however, for programming simplicity a 2D model will be considered during the reliability analysis. Here, APDL commands implemented in Mechanical ANSYS software are used to integrate the reliability process and variable boundaries. An illustration of the studied stem with both bone layers is shown in Fig. 1b (dimensions in millimeters)

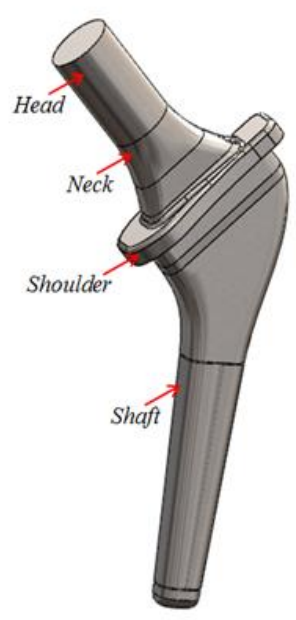

$a$

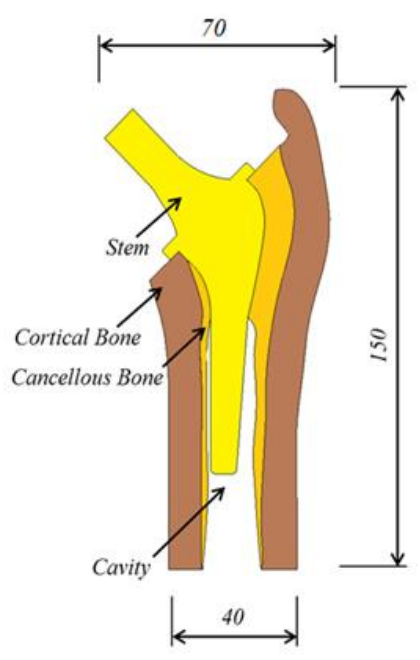

$b$
Fig. 1. Illustration of a) 3-dimensional stem model and b) 2-dimensional model of stem with both cortical and concellous layers.

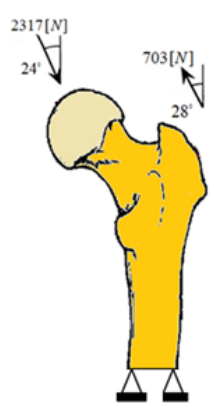

$a$
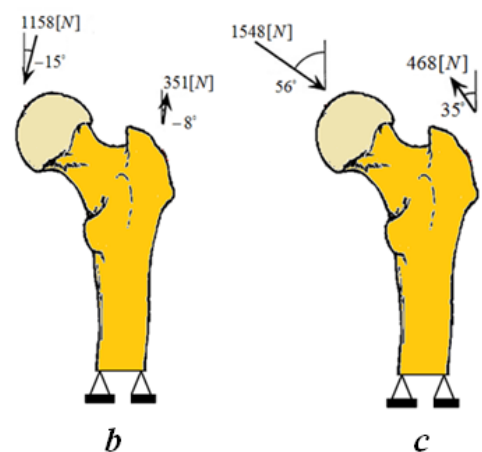

c
Fig. 2. a) One-legged stance case (L1), b) Abduction case (L2), and c) Adduction case (L3).

\section{B. Boundary conditions}

For the studied case, three representative daily loading conditions of one-legged stance (L1), extreme ranges of motion of abduction (L2), and adduction (L3) are considered [6], as shown in Fig. 2. The boundary conditions at the distal end have no effect on the stresses in the proximal region. The fixation is carried out on lower bone cut (on the cortical layer) to avoid rigid-body motion

\section{Mechanical properties}

It is well-known that bone tissue possesses a complicated microstructure. The bone also adapts to the actual loading and many other factors and is therefore in general both heterogeneous and anisotropic. Density information for the cancellous bone was then used to estimate the stiffness through an empirical relationship between density and Young's modulus given by Rho et al. [7]. This resulted in a non-homogeneous distribution of stiffness ranging from 1 to 5.6GPa for the cancellous bone. In Reference [8], an average of the reported stiffness values of the diaphysis and metaphysis were used for the entire cortical region resulting in stiffness values of $19.7,13.1$ and $10.4 \mathrm{GPa}$ in the axial, circumferential and thickness directions respectively [9]. In the present analysis, the material properties of bone have for simplicity been assumed as being linearly elastic and isotropic. A new formulation relating the yield stress and elasticity will be developed in the next section.

\section{Proposed mathematical model}

The mechanical properties of bone depend on its composition and structure. The most representative compositional variable is the ash density [5]. The Young's modulus against the ash density relationship can be written as follows:

$$
E=10500 \rho_{\alpha}^{2.57 \pm 0.04}
$$

The yield stress against the ash density relationship can be written as follows:

$$
\sigma_{C}=117 \rho_{\alpha}^{1.93 \pm 0.04}
$$

where $\rho_{\alpha}$ is the ash density. This expression explains over $96 \%$ of the statistical variation in the mechanical behaviour of combined vertebral and femoral data over the range of ash density $\left(\rho_{\alpha}=\left(0.03 \div 1.22 \mathrm{~g} / \mathrm{cm}^{3}\right)\right)$. After having simple developments, a generalized relationship between the yield stress in compression and the Young's modulus can be written as follows:

$$
\sigma_{C}=117\left(\frac{E}{10500}\right)^{\frac{1.93+0.04}{2.57 \pm 0.04}}
$$

The ultimate compressive strength of the bone structure is bigger than its ultimate tension one. Thus, different values have been used for a tension/compression ratio, from 0.5 to 0.7 for cortical bone and from 0.7 to 1 for cancellous bone [10]. 


\section{STRATEGY AND ALGORITHM}

\section{A. Reliability analysis}

In structural reliability theory, very effective techniques have been developed during the last 40 years to estimate the reliability, namely first order reliability methods (FORM), second order reliability methods (SORM) and simulation techniques [4]. The reliability problem can be performed for a single failure mode and for multiple failure ones.

For a single failure mode case, reliability index $\beta$ is evaluated by solving a constrained minimization problem [11]:

$$
\beta=\min d \mathbf{1}^{-} \text {, subject to: } H \mathbf{a}^{-}=0
$$

The solution to problem (12) defines the Most Probable failure Point (MPP) and the resulting minimum distance between the limit state function $H(\mathbf{u})=0$ and the origin, is called reliability index $\beta$. In Figure (3), the reliability index levels are represented by ellipses in the physical space and by circles in the normalized space. The mean value is represented by $m_{i}$ in the physical space, but it is considered as the origin of the normalized space.

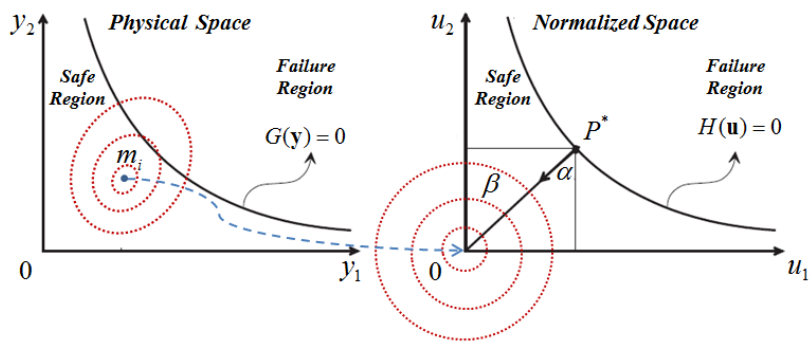

Fig. 3. Transformation from physical space to normalized one for a single failure mode.

For a multiple failure mode case, multiple conflicting criteria are involved in evaluating a complicated design system, several constraint functions. This way the problem can be written as follows:

$$
\beta=\min d \mathbf{1}^{-} \text {subject to: } \mathbf{H} \mathbf{1}=\mathbf{0}
$$

where $\mathbf{H} \mathbf{1}^{-}=\mathbf{0}$ is the vector of limit state functions. Figure 4 shows an example with two normalized variables $u_{1}$ and $u_{2}$ with a design point $P^{*}$ (or MPP) located at the intersection of the two limit state functions $H_{1} \mathbf{Q}_{=}=0$ and $H_{2} \mathbf{q}=0$. The global optimum solution in the normalized space provides us with the minimum distance between the intersection point and the origin. The genetic algorithms lead to global optimum solution but they necessitate a high computing time. Thus there is a strong motivation to develop a new optimization algorithm in order to provide a global optimum with a reasonable computing time. In next section, a special reliability algorithm is elaborated considering the developed mathematical formulation of the compression strength against the Young's modulus relationship.

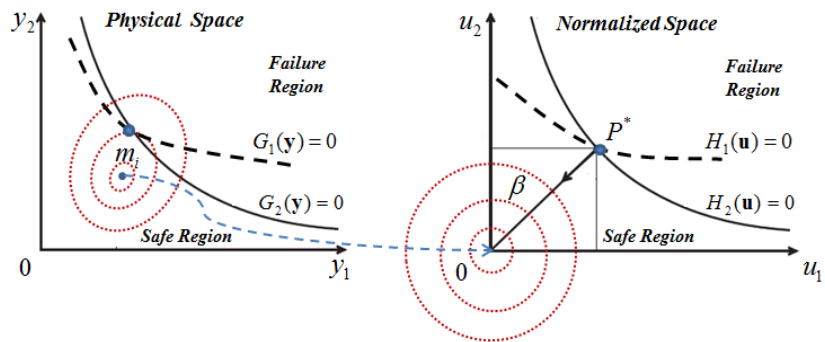

Fig. 4. Transformation from physical space to normalized one for a multiple failure mode.

The probability of failure is classically evaluated by numerical integral methods that lead to a high computing time [4]. However, using FORM approximation, the probability of failure is simply evaluated by:

$$
P_{f} \approx \Phi(-\beta)
$$

where $\Phi(\bullet)$ is the standard Gaussian cumulated function given as follows:

$$
\Phi(Z)=\frac{1}{\sqrt{2 \pi}} \int_{-\infty}^{Z} e^{-\frac{z^{2}}{2}} d z
$$

For practical engineering, Eq. (15) gives sufficiently accurate estimation of the failure probability [12]. Following Hasofer and Lind [11], it is preferable to work in a standard normalized space of independent Gaussian variables rather than in the space of physical variables. Hence, we simply adopt the law for a normal distribution, and define a normalized variable $u_{i}$ by the transformation:

$$
u_{i}=\frac{y_{i}-m_{i}}{\sigma_{i}}
$$

where $y_{i}$ is a random variable with the mean value $m_{i}$ and the standard-deviation $\sigma_{i}$. Here, the mean value $m_{i}$ may be adopted as a design variable $x_{i}$. When considering several random variables, the results are subject to classical difficulties in nonlinear programming: existence of local minima, gradient approximation and computational time. Since the reliability analysis is carried out in a normalized space, a special technique based on zero order methods (curve 
fitting method) is developed in order to deal with the particular form of the reliability problem with variable failure modes.

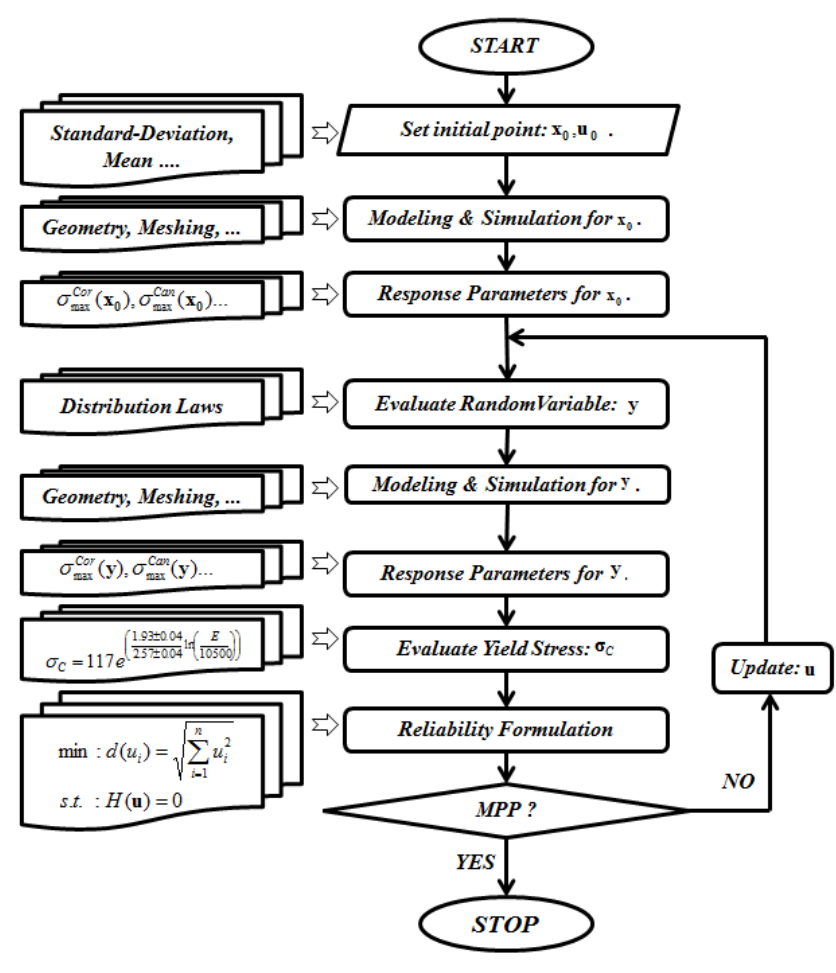

Fig. 5. Flowchart of reliability algorithm.

\section{B. Proposed reliability algorithm}

The implemented reliability algorithm is realized using Mechanical ANSYS software with APDL (ANSYS Parametric Design Language) technology. The developed algorithm (Figure 5) consists of the following steps:

1. Initialize the probabilistic model $\left(\mathbf{x}_{\mathbf{0}}, \mathbf{u}_{\mathbf{0}}\right)$ : mean values, standard-deviations, normalized vector ...,

2. Perform modeling and simulation to provide the structural response parameters for the mean values $\mathbf{x}_{\mathbf{0}}$,

3. Evaluate the random vector and re-simulate to get the structural response parameters for the random variables $\mathbf{y}$,

4. Use Equation 3 to estimate the corresponding compression strength $\sigma_{C}$,

5. Apply Equation 4 to estimate the reliability index $\beta$,

6. Stop if converged for MPP, else update the normalized vector $\mathbf{u}$ and go to 3 .
At each iteration, a new design space is created because of the correlation between the constraint functions and the optimization variables. In addition, for the multiple failure modes, Steps 4 to 6 necessitate more computing time in order to find the intersection point.

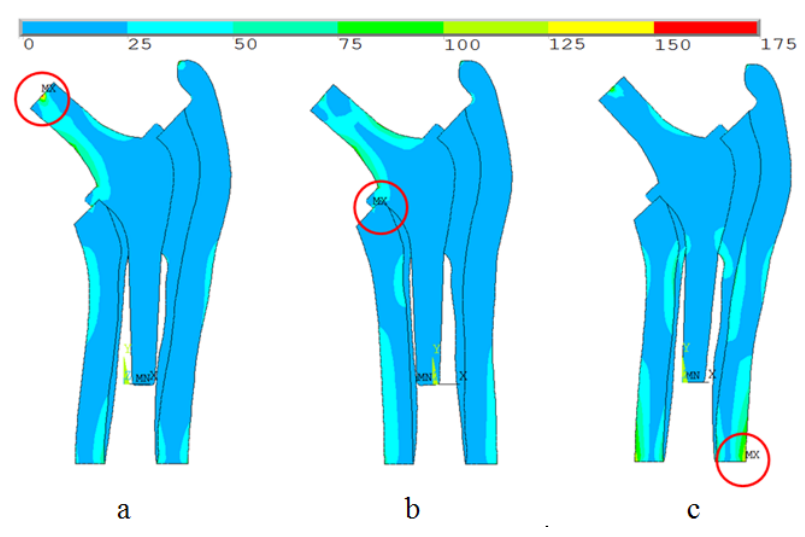

Fig. 6. von-Mises stress of MPP for the mean point when applying the three loading cases a) L1, b) L2, and c) L3.

\section{NUMERICAL RESULTS}

\section{A. Problem description}

The objective is to find the most probable failure point (MPP) by minimizing the distance between the limit state function and the origin of the normalized space. The Young's modulus and Poisson's ratio of the three material components $E_{C o r}, E_{C a n}, E_{M e t}, v_{C o r}, v_{C a n}, v_{M e t}$ are considered as uncertain parameters. Their mean values $m_{E_{C o r}}, m_{E_{C a n}}, m_{E_{M e t}}, m_{v_{C o r}}, m_{E_{G o r}} m_{E_{C o r}}$ are presented in Table 1. The thickness of this academic 2D model is considered constant $(T=10 \mathrm{~mm})$. The number of elements considered for optimization is 1476 nonlinear elements (8-node/PLANE82) and the total number of nodes is 4825 nodes. The cortical (or compact) bone part is assumed to be a homogeneous and isotropic material with Young's modulus $E=19.7 \mathrm{GPa}$ and Poisson's ratio $v=0.33$. The used number of elements for cortical region is 699 elements. The cancellous (or spongy) bone layer was also assumed to be a homogeneous and isotropic material with Young's modulus $E=5.6 \mathrm{GPa}$ and Poisson's ratio $v=0.33$. The used number of elements for cancellous region is 220 elements. However, the marrow part was regarded as a cavity, and neglected in finite element analysis [13]. Titanium alloy is considered to be the best bio-compatible material among all other prosthesis materials. So, the femoral stem material is considered to be made of titanium alloy (Ti-6Al-4V). Titanium-based alloys have a typical Young's modulus range of 100 to $120 \mathrm{GPa}$ [14]. The material properties for the proximal femoral hip stem considered for the analysis are Young's modulus $E=120 \mathrm{GPa}$, Poisson's ratio: $v=0.36$. The corresponding yield strength: $\sigma_{y}=900 \mathrm{MPa}$ [15]. The corresponding compression strengths $\left(\sigma_{C}^{c o r}\right.$ and $\sigma_{C}^{c a n}$ ) for both bone layers (cortical and 
cancellous layers) are calculated according to Equation 3 (see Table 1). Figure 6 shows the von-Mises stress distributions for the three daily loading cases.

After having performed a direct simulation, the resulting von-Mises stress values for the different layers and for the three studied loading cases: L1, L2 and L3, are shown in Table 1 .

\section{B. Reliability results}

The reliability assessment is carried out considering that random variables $y_{i}$ corresponding to the Young's modulus and the Poisson's ratio of different layers $\left(E_{C o r}, E_{C a n}, E_{M e t}, v_{C o r}, v_{C a n}, v_{M e t}\right)$ are normally considered distributed, their mean values are presented in Table 1, and their standard deviations are proposed proportional to the mean values (20\%). In our previous structural studies [4], the proportionality value is considered as $10 \%$, but in order to take in account the tension strength, it is doubled. Considering the von-Mises stress as a fracture indicator, the reliability analysis is carried out for a single failure mode and for multiple failure ones.

TABLE I. MATERIAL PROPERTIES AND VON-MISES STRESS DISTRIBUTION FOR THE MEAN POINT

\begin{tabular}{|c|c|c|c|}
\hline Parameters & L1 & L2 & L3 \\
\hline$m_{E_{\text {cor }}}(\mathrm{MPa})$ & \multicolumn{3}{|c|}{19700} \\
\hline$m_{E_{2}}(\mathrm{MPa})$ & \multicolumn{3}{|c|}{5600} \\
\hline$m_{E_{3}}(\mathrm{MPa})$ & \multicolumn{3}{|c|}{120000} \\
\hline$m_{v_{1}}$ & \multicolumn{3}{|c|}{0.33} \\
\hline$m_{v_{2}}$ & \multicolumn{3}{|c|}{0.33} \\
\hline$m_{v_{3}}$ & \multicolumn{3}{|c|}{0.36} \\
\hline$\sigma_{\max }^{\text {Metal }}(\mathrm{MPa})$ & 167.96 & 103.56 & 113.33 \\
\hline$\sigma_{\max }^{\text {Cor }}(\mathrm{MPa})$ & 129.34 & 90.32 & 143.97 \\
\hline$\sigma_{C}^{\text {Cor }}(\mathrm{MPa})$ & {$[184.53 \div 190.97]$} \\
\hline$\sigma_{\max }^{\text {Can }}(\mathrm{MPa})$ & 44.30 & 23.47 & 62.08 \\
\hline$\sigma_{C}^{\text {Can }}(\mathrm{MPa})$ & \multicolumn{2}{|c|}{$[71.72 \div 74.22]$} \\
\hline
\end{tabular}

Single failure mode case: According to Table 2, the most probable failure scenario may be occurred on the cortical layers for the first and the second loading cases (L1 and L2). However, the most probable failure scenario may be occurred on the cancellous bone when applying the third loading case (L3).
TABLE II. MPP FOR SINGLE FAILURE MODE

\begin{tabular}{|c|c|c|c|}
\hline Parameters & LI & $L 2$ & L3 \\
\hline$E_{1}(\mathrm{MPa})$ & 12039 & 7447.1 & 14438.4 \\
\hline$E_{2}(\mathrm{MPa})$ & 3404.4 & 1313.9 & 5302.6 \\
\hline$E_{3}(\mathrm{MPa})$ & 119030 & 105910 & 118556 \\
\hline$v_{1}$ & 0.3286 & 0.3185 & 0.3207 \\
\hline$v_{2}$ & 0.3292 & 0.3027 & 0.3243 \\
\hline$v_{3}$ & 0.3406 & 0.3010 & 0.3339 \\
\hline$\sigma_{\max }^{\text {Metal }}(\mathrm{MPa})$ & 167.82 & 113.05 & 113.11 \\
\hline$\sigma_{\max }^{C o r}(\mathrm{MPa})$ & $\underline{129.35}$ & $\underline{90.27}$ & 141.11 \\
\hline$\sigma_{C}^{C o r}(\mathrm{MPa})$ & $\begin{array}{l}{[129.18,} \\
130.15]\end{array}$ & $\begin{array}{c}{[89.54,} \\
91.23]\end{array}$ & $\begin{array}{c}{[147.35,} \\
149.93]\end{array}$ \\
\hline$\sigma_{\max }^{\text {can }}(\mathrm{MPa})$ & 44.25 & 22.18 & 68.89 \\
\hline$\sigma_{C}^{C a n}(\mathrm{MPa})$ & $\begin{array}{c}{[48.68,} \\
51.76]\end{array}$ & $\begin{array}{c}{[23.19,} \\
25.98]\end{array}$ & $\begin{array}{c}{[68.73,} \\
71.34]\end{array}$ \\
\hline$\beta$ & 2.77 & 5.05 & 1.42 \\
\hline$P_{f}$ & $2.80 \times 10^{-3}$ & $2.21 \times 10^{-7}$ & $7.78 \times 10^{-2}$ \\
\hline
\end{tabular}

Multiple failure mode case: According to Table 3, the probability of failure when considering multiple failure scenarios is smaller than the MPP when considering the single failure mode.

TABLE III. MPP FOR MULTIPLE FAILURE MODE

\begin{tabular}{|c|c|c|c|}
\hline Parameters & $\boldsymbol{L 1}$ & $\boldsymbol{L 2}$ & $\boldsymbol{L 3}$ \\
\hline$E_{C o r}(\mathrm{MPa})$ & 12083 & 7481.8 & 13420 \\
\hline$E_{C a n}(\mathrm{MPa})$ & 2703 & 1241.6 & 5308.2 \\
\hline$E_{M e t}(\mathrm{MPa})$ & 117060 & 101480 & 118250 \\
\hline$v_{C o r}$ & 0.3298 & 0.3289 & 0.32216 \\
\hline$v_{C a n}$ & 0.3296 & 0.3006 & 0.32162 \\
\hline$v_{M e t}$ & 0.3413 & 0.3005 & 0.32435 \\
\hline$\sigma_{\max }^{M e t a l}(\mathrm{MPa})$ & 167.83 & 113.91 & 113.03 \\
\hline$\sigma_{\max }^{\text {Cor }}(\mathrm{MPa})$ & $\underline{\mathbf{1 2 9 . 3 4}}$ & $\underline{\mathbf{9 0 . 3 1}}$ & $\underline{\mathbf{1 4 0 . 5 0}}$ \\
\hline$\sigma_{C}^{\text {Cor }}(\mathrm{MPa})$ & {$[129.52$,} & {$[89.86$,} & {$[139.75$,} \\
\hline$\sigma_{\max }^{\text {Can }}(\mathrm{MPa})$ & $\underline{\mathbf{4 2 . 1 7}}$ & $\underline{\mathbf{2 2 . 1 9}}$ & $\underline{\mathbf{7 0 . 9 5}}$ \\
\hline
\end{tabular}


TABLE III. CONT.

\begin{tabular}{|c|c|c|c|}
\hline$\sigma_{c}^{\text {can }}(\mathrm{MPa})$ & $\begin{array}{c}{[40.67,} \\
43.79]\end{array}$ & $\begin{array}{c}{[22.19,} \\
24.93]\end{array}$ & $\begin{array}{c}{[68.79,} \\
71.39]\end{array}$ \\
\hline$\beta$ & 3.24 & 5.12 & $\mathbf{1 . 7 0}$ \\
\hline$P_{f}$ & $5.98 \times 10^{-4}$ & $1.53 \times 10^{-7}$ & $\mathbf{4 . 4 6} \times 10^{-2}$ \\
\hline
\end{tabular}

\section{Discussion}

Since the reliability analysis is carried out in a normalized space, a special technique is developed in order to perform the particular form of the reliability problem using APDL (ANSYS Parametric Design Language). The optimization algorithm, which is illustrated in Fig. 5, supplies us all information about the objective and constraint functions. A developed mathematical formulation is also integrated to this algorithm that leads to iterative changes of design space. Its capability to provide a robust solution leads to good convergence stability. In addition, to improve our design, a significant increase of the standard-deviation values is considered during the optimization process. The developed algorithm is applied on three different daily loading cases. The von-Mises stress values are considered as fracture indicators. This way the failure scenarios are related to the fracture of one or several components. The resulting maximum stress values are located in different layers. For the first and second loading cases, the resulting maximum stress values are found in the metallic stem. However, for the third loading case, it is located in the cortical bone (Table 1). From point of view reliability, all fracture indicators are subjected to be changed because of the uncertainty of material properties. For a single failure scenario, the most probable failure point (MPP) is found at $\beta=1.42$ that leads to a level of probability of failure: $P_{f}=7.78 \%$ (Equations $6 \& 7$ ). The failure may occur in the cancellous bone layers. In contrast, the most probable failure point for the first and second loading cases (L1 and L2) may occur at the cortical bone layer. This leads to higher reliability levels than the third loading case (L3). Thus, the second case is the most reliable case $(\beta=5.05)$ with a very small probability of failure. Furthermore, when considering a multiple failure scenarios, the intersection point of both cortical and cancellous layers is found at a farther distance ( $\beta=1.70)$ than the MPP corresponding to the single failure mode. Thus, the probability of failure of the third loading case is worse than the first and second loading cases. In the literature, several works correlate mechanical properties of bone materials with its composition [10]. In this work, we propose using a formulation that directly correlates the mechanical properties of bone materials in order to apply the reliability concept. This way the Young's modulus represents the resistance and the yield stress represents the limit state (scenario of failure). The developed algorithm can be considered as an efficient tool for reliability assessments in prosthesis design. It leads to reliable results and can be extended to different material behavior cases. It also has a good efficiency in finding the intersection point when considering multiple failure scenarios.

\section{CONCLUSION}

When considering the uncertainty (or randomness) on the bone material properties, the change of these properties leads to change of its resistance. For example, when changing the elasticity modulus of the bone, the corresponding yield strength will be changed. Thus, the uncertainty should be considered when designing prostheses. This work essentially consists in integrating reliability analysis into prosthesis design. Here, a development of an equation for yield stress against elasticity modulus relationship is carried out. The proposed equation based on Keller's model is next integrated to the developed reliability algorithm. Furthermore, since the ultimate compressive strength of the bone structure is bigger than its ultimate tension one, the standard-deviation values are doubled. The convergence robustness and stability of the developed algorithm is demonstrated on single and multiple failure modes. To conclude, the developed algorithm automatically correlates the mechanical properties of bone materials using the reliability philosophy. It can be considered as an efficient tool for reliability assessments, especially when comparing with the robustness of the genetic algorithms. For future developments of this algorithm, the different bone behaviors (orthotropic, anisotropic ...) can be considered in order to get realistic results. It could be very interesting to directly relate the developed algorithm to CT-scan data (CT grey value of the bone in terms of Hounsfield units (HU)). This integration then allows us finding the optimum position of the implant relative to bone layers with object of insuring a required reliability level.

\section{References}

[1] C.D. Gonzalez, A. New, M. Browne, "Probabilistic finite element analysis of the uncemented hip replacement-effect of femur characteristics and implant design geometry," J. of Biomechanics, 43 (3), pp. 512-520, 2010.

[2] S.A. Shaik, K. Bose, H.P. Cherukuri, "A study of durability of hip implants," J. Materials and Design, 42, pp. 230-237, 2012.

[3] J.L. McGinley, R. Baker, R. Wolfe, M.E. Morris, "The reliability of three-dimensional kinematic gait measurements: a systematic review," Gait \& Posture, 29 (3), pp. 360-369, 2009.

[4] G. Kharmanda, I. Antypas, "Integration of Reliability Concept into Soil Tillage Machine Design," J. of Don State Technical University, Vestnik of DSTU, 15 (2), pp. 22-31, 2015.

[5] T.S. Keller, "Predicting the compressive mechanical behavior of bone," J. of Biomech., 27, pp. 1159-1168, 1994.

[6] G.S. Beaupré, T.E. Orr, D.R. Carter, "An approach for time-dependent bone modeling and remodeling-application: A preliminary remodeling simulation," J. of Orthopaedic Research, 8 (5), pp. 662-670, 1990.

[7] J.Y. Rho, R.B. Ashman, C.H. Turner, "Young's modulus of trabecular and cortical bone material: Ultrasonic and micro tensile measurements," J. of Biomechanics, 26 (2), pp. 111-119, 1993.

[8] E. Cheal, M. Spector, W. Hayes, "Role of loads and prosthesis material properties on the mechanics of the proximal femur after total hip arthroplasty," J. of Orthopaedic Research 10 (3), 1992, pp. 405-422. 
[9] M.G. Joshi, S.G. Advani, F. Miller, M.H. Santare, "Analysis of a femoral hip prosthesis designed to reduce stress shielding," J. of Biomech., 33, pp. 1655-1662, 2002.

[10] M. Doblare, J.M. Garcia, M.J. Gomez, "Modelling bone tissue fracture and healing: A review," Engineering Fracture Mechanics, 71, pp. 18091840, 2004.

[11] A.M. Hasofer, N.C. Lind, "An exact and invariant first order reliability format,” J. Eng. Mech, ASCE, EM1, 100, pp. 111-121, 1974.

[12] B.M. Ayyub, R.H. McCuen, Probability, Statistics and Reliability for Engineers and Scientists, CRC Press, Taylor \& Francis Group, 2011.
[13] K. Tsubota, T. Adachi, Y. Tomita, "Functional adaptation of cancellous bone in human proximal femur predicted by trabecular surface remodeling simulation toward uniform stress state," J. of Biomech., 35, pp. 1541-1551, 2002.

[14] B. Bougherara, R .Zdero, S. Shah, M. Miric, M. Papini, P. Zalzal, E.H. Schemitsch, "A biomechanical assessment of modular and monoblock revision hip implants using FE analysis and strain gage measurements," J. of Orthopaedic Surgery and Research, 5:34, 2010.

[15] L.D. Zardiackas, M.J. Kraay, H.L. Freese, "Titanium, Niobium, Zirconium, and Tantalum for Medical and Surgical Application," American Society for Testing and Materials (ASTM), Ann Arbor, 2006. 